\title{
O. Univ.-Prof. Dipl.-Ing. Dr. techn. Rudolf Dutter als Wissenschaftler, Kollege und Persönlichkeit
}

Rudolf Dutter wurde am 11. November 1946 in Grünsbach, Niederösterreich geboren. Er besuchte die Volksschule in Grünau, die Hauptschule in Obergrafendorf und die Höhere Technische Bundeslehranstalt in St. Pölten. Anschließend studierte er an der Technischen Hochschule Wien „Technische Mathematik“. Schon seine Diplomarbeit mit dem Thema „Eine Anwendung der Wartezeitentheorie auf den Verkehrsfluss bei Fahrbahnverengungen" verriet sein Interesse für die Statistik.

Danach zeigte sich seine bemerkenswerte Flexibilität, da er nach Abschluss des Diplomstudiums den Aufbruch nach Nordamerika wagte und sein Doktoratsstudium an der Université de Montréal in Kanada aufnahm. Dies machte ihn als Dissertant von Prof. A. Joffe und Prof. I. Guttman auch mit Bayes'schen Ideen der Statistik bekannt und er erwarb ein heute auch in Österreich aktuelles Ph.D. mit einer Dissertation über „A Discussion of the Handling of Outliers to Detect Spuriousness in the General Univariate Model, Full and Non-Full Rank Cases“.

Die wissenschaftlichen Erfahrungen in Kanada führten ihn zu einem der renommiertesten Statistiker des 20. Jahrhunderts, Prof. Peter J. Huber, der damals an der ETH-Zürich wirkte. Dort war Herr Dr. Dutter als Assistent von 1. Oktober 1973 bis 30. September 1976 in der Fachgruppe für Statistik tätig. Dabei entwickelte er eine Faszination für „Computational Statistics“, die bis heute anhält. So schrieb er unter anderem Computerprogramme für robuste statistische Schätzverfahren.

Im Jahre 1976 zog es ihn nach Österreich zurück und er wurde Universitätsassistent am Institut für Statistik der TU Graz bei Prof. U. Dieter. Dort habilitierte er sich im Jahre 1980 für das Fach „Statistik“ und arbeitete als Universitätsdozent am selben Institut bis zum Jahre 1984.

Inzwischen war die Bedeutung der Computer für die Statistik allgemein erkannt worden und die Besetzung der Nachfolge von Prof. Walther Eberl am Institut für Statistik und Wahrscheinlichkeitstheorie der Technischen Universität Wien trug dem Rechnung. Daher wurde Herr Dozent Dutter im Jahre 1984 auf das umgewidmete Ordinariat für Technische Statistik berufen. Er trat seinen Dienst am 1. Juli des selben Jahres an der Technischen Universität Wien an und übersiedelte mit seiner Familie nach Wien um sich seiner neuen Aufgabe voll und ganz zu widmen. Dies tat er mit Begeisterung und der Aufbau einer zeitgemäßen computergestützten Statistik war eine beträchtliche Arbeit, die Herr Prof. Dutter mit Elan bewältigte. Dabei entstand schon in Graz beginnend ein Analysesystem genannt „DAS“, das laufend verbessert und erweitert wurde.

Prof. Dutter förderte auch die Arbeit seiner Assistenten am frei verfügbaren Analysesystem „R“ und seine Mitarbeiter waren zum Teil im Kernentwicklungsteam von „R“ tätig. Einige seiner Assistenten sind inzwischen selbst zu Professoren, auch an anderen Universitäten, arriviert.

Herr Kollege Dutter hat eine soziale Ader. Ich erinnere mich daran, dass er einmal ein Kind aus Rumänien in seine Familie aufnahm. Er hat zwei Töchter und zwei Enkelkinder und ist ein begeisterter Großvater.

Neben seinen beruflichen Aktivitäten ist Herr Prof. Dutter auch sportlich sehr aktiv und hat bis vor kurzem sogar Marathonläufe erfolgreich bewältigt. Seine Schilderung des 
Bergmarathon in der Schweiz lässt die Grenzen der Leistungsfähigkeit des Menschen erkennen.

Da Herr Prof. Dutter von 1997 bis zum Jahre 2003 Herausgeber der Österreichischen Zeitschrift für Statistik war, entstand die Idee, ihm dafür als kleinen Dank ein Sonderheft zu widmen. Seine unprätentiöse Art, die in einer Zeit von PR-Überfluss sehr sympathisch ist, lässt dies angemessen erscheinen.

Seit über einem Vierteljahrhundert bin ich nun gemeinsam mit Rudi Dutter am Institut tätig, und obwohl unsere Forschungsinteressen verschieden sind, fanden wir bald private Gemeinsamkeiten. Dafür bin ich dankbar und hoffe, dass auch er dies so empfindet.

Die unermüdliche Arbeitskraft und der Einsatz für das Fach Statistik von Herrn Prof. Dutter sowie seine menschlichen Ansichten haben ihn zu einem geschätzten Kollegen und durch private Kontakte auch zu einem Freund werden lassen. Ihm und seinen Lieben wünsche ich alles Gute für die Zukunft.

Reinhard Viertl 